\title{
EVALUACIÓN DE ENSAYOS DE PROGENIES DEL PROGRAMA DE MEJORAMIENTO DE Eucalyptus globulus Labil. PARA RESISTENCIA A SEQUÍA
}

\author{
Rojas, Patricio ${ }^{3}$; Ipinza, Roberto ${ }^{4}$ \\ Molina, María Paz y Gutiérrez, Braulio
}

\section{RESUMEN}

Debido a las excepcionales propiedades de su fibra, Eucalyptus globulus Labill., "Tasmanian Blue Gum", es la principal especie para la producción de pulpa y papel en el mundo. En Chile existen más de 500 mil hectáreas plantadas con esta especie, lo que representa más de un quinto del total de plantaciones en el país. La especie fue introducida a Chile a fines del siglo XIX para ser usada como postes en la minería del carbón en Lota (región del Biobío), posteriormente como consecuencia de exitosos ciclos de selección y propagación se ha dado origen a una raza local que ha experimentado una adecuada adaptación a distintas condiciones de clima en el país.

En los años 90 varias organizaciones chilenas dieron inicio a programas para mejorar la resistencia al frío, el crecimiento y la calidad de la fibra de esta especie, pero no consideraron la resistencia a la sequía como un carácter de valor comercial. Sin embargo, resultados de investigaciones del Instituto Forestal (INFOR) muestran que el cambio climático disminuirá la productividad de las plantaciones de eucalipto en alrededor de 6 a $8 \%$ en la zona centro centro-sur de Chile, debido a la reducción de las precipitaciones y el aumento de las temperaturas.

En 2006 INFOR inició un proyecto de investigación para las zonas semiáridas de Chile, orientado a seleccionar, recombinar y propagar genotipos de E. globulus resistentes a la sequía. Para este fin se consideraron tres poblaciones: (i) Genotipos selectos por volumen y representados en un huerto semillero clonal, correspondientes al programa de mejora genética de INFOR; (ii) Familias de polinización abierta de 107 árboles plus seleccionados en condiciones de aridez; y (iii) Familias de polinización controlada generadas por el cruzamiento de madres de la población (i) con polen de la población (ii). Este último material fue concebido para combinar la superioridad genética de los clones del huerto semillero con la resistencia a la sequía de la raza local chilena de E. globulus.

En el presente documento se entregan y discuten los resultados de dos ensayos de progenies de polinización abierta y uno de polinización controlada, compuestos por material de las poblaciones mencionadas, que fueron establecidos entre los años 2009 y 2010 en sitios representativos de condiciones de aridez en la región del Biobío. Los parámetros genéticos para tolerancia a sequía de E. globulus se obtuvieron a partir de la supervivencia y crecimiento de 24 familias de polinización controlada y 61 familias de polinización abierta de 5 a 6 años de edad, considerando como controles a lotes de semilla comercial de E. globulus y a otras especies de eucaliptos reconocidas por su tolerancia a la sequía, particularmente $E$. camaldulensis y $E$. cladocalyx.

Se discute la posibilidad de aplicación de esta estrategia para mejorar la tolerancia a la sequía de E. globulus en las zonas áridas de la región del Biobío y se incluye la selección de las mejores familias de polinización abierta y controlada para desarrollo comercial.

Palabras clave: Mejoramiento genético, Eucalyptus globulus, Tolerancia a sequía

\footnotetext{
${ }^{3}$ MSc. Ingeniero Forestal. Instituto Forestal, Sede Metropolitana. parojas@infor.cl

${ }^{4}$ Dr. Ingeniero de Montes. Instituto Forestal, Sede Los Ríos. roberto.ipinza@infor.cl

5 MSc. Ingeniero Forestal. Instituto Forestal, Sede Bíobío. mmolina@infor.cl

${ }^{6}$ Ingeniero Forestal. Instituto Forestal. Sede Bíobío. bgutierr@infor.cl
} 


\section{SUMMARY}

Eucalyptus globulus Labill "Tasmanian Blue Gum" is the main species for pulp and paper production in the world due to its exceptional fiber properties. There are over than 500 thousand 13 hectares planted with the species in Chile and that represents more than $23 \%$ of the total planted forests in the country. The species was introduced by the end of XIX century for coal mining poles in Colcura (Bío Bío Region) and due to successive selection and propagation cycles a "Chilean Land Race" was formed which has been well adapted to dry climatic conditions in the country.

By 1990 many organizations in Chile started breeding programs to improve frost hardiness, growth, yield and fiber quality of the species, but drought hardiness was not included as a commercial trait. Last INFOR survey results shows that the climatic change will decrease the plantations productivity due to lower precipitation and higher temperatures in the central and south region of Chile up to $6-8 \%$.

An INFOR research project started on 2006 was oriented to select, recombine and propagate $E$. globulus drought hardiness genotypes in the Chilean semiarid region. Three populations were included for this purpose: $(A)$ Selected genotypes ranked by volume in a clonal seed orchard that correspond to the INFOR tree breeding program, (B) Open pollinated families of 107 plus trees selected on extreme dry conditions, and (C) Control pollinated families generated by intraspecific crosses of population $(A \times B)$. This material combined the clonal orchard genotypes genetic superiority and the E. globulus Chilean land race drought hardiness.

This paper shows the results of two open-pollinated trials and one controlled pollination trial from these three populations planted in representative sites of dry land areas of the Bío-Bío Region on 2009-2010. Genetic parameters for E. globulus drought tolerance were obtained through the survival and growth of 24 control pollinated families and 61 open pollinated families at 5-6-yearold using as controls commercial seed sources of $E$. globulus and drought tolerant species like $E$. camaldulensis and E. cladocalyx.

Conclusion are related to the applicability of this breeding strategies for improving $\mathrm{E}$. globulus drought tolerance in dry zones areas of the Bío-Bío Region including selection of best CP and OP progenies for commercial deployment.

Keywords: Breeding, Eucalyptus globulus, drought tolerance 


\section{INTRODUCCIÓN}

Debido a las excepcionales propiedades de su fibra, Eucalyptus globulus Labill (Tasmanian Blue Gum) es la principal especie para la producción de pulpa y papel en el mundo. En Chile existen 563.813 hectáreas plantadas con esta especie, lo que representa un $23 \%$ del total de plantaciones exóticas en el país. La especie fue introducida a Chile a fines del siglo XIX para ser usada como postes en la minería del carbón en Colcura (región del Biobío), posteriormente como consecuencia de exitosos ciclos de selección y propagación se ha dado origen a una raza local que ha experimentado una adecuada adaptación a distintas condiciones de clima en el país.

En los años 90 varias organizaciones chilenas dieron inicio a programas para mejorar la resistencia al frío, el crecimiento y la calidad de la fibra de esta especie, pero no consideraron la resistencia a la sequía como un carácter de valor comercial. Sin embargo, resultados de investigaciones de INFOR muestran que el cambio climático disminuirá la productividad de las plantaciones de eucalipto en alrededor de 6 a $8 \%$ en la zona centro centro-sur de Chile, debido a la reducción de las precipitaciones y el aumento de las temperaturas.

E. globulus crece en forma natural en el sudeste de Australia, donde conforma un complejo de especies junto con E. bicostata, E. maideni y E. pseudoglobulus, las cuales difieren básicamente en el número y tamaño de yemas florales por umbela (Dutkowsky y Potts, 1999). Estudios realizados sobre su resistencia a la sequía muestran que existe una significativa variación genética, tanto en su distribución natural como en los resultados de ensayos de progenies plantados en Australia (Dutkowsky, 1995) y España (Toro et al., 1998). La especie se encuentra genéticamente estratificada en 13 razas y 20 sub-razas, para las cuales estudios de marcadores moleculares han demostrado que tres líneas genéticas conforman la población principal: (a) Victoria; (b) King Island y Oeste de Tasmania; y (c) Este de Tasmania e Islas Furneaux (Dutkowsky et al., 1997). Potts y Dutkowsky (2012) concluyen que las procedencias de E. globulus más vulnerables a sequía son las de King Island y las de la costa oeste de Tasmania, mientras que las más tolerantes son aquellas de Jeerelang y el norte de Tasmania. Estos últimos orígenes han resultado ser una adecuada fuente de semillas de $E$. bicostata para condiciones de alta restricción hídrica en Chile.

Una de las innovaciones tecnológicas para la polinización controlada de E. globulus fue la aplicación de la técnica de polinización en un paso, o en una visita, desarrollada paralelamente en Chile (Espejo et al., 1995) y Australia (Williams et al., 1999), a partir de investigaciones previas efectuadas en E. gunnii por Cauvin (1988). El comparativamente bajo costo de esta técnica permitió obtener semilla de polinización controlada a escala operacional en huertos semilleros clonales (Rojas y Griffin, 1997; Rojas et al., 2001)

El proyecto "Generación y producción de plantas de Eucalyptus globulus tolerantes a la sequía”, financiado por INNOVA Chile de CORFO y ejecutado conjuntamente por el Instituto Forestal (INFOR) y el Centro Regional de Investigación La Platina del Instituto de Investigación Agropecuaria (INIA), tuvo por objetivo generar disponibilidad para los propietarios y empresas forestales de las zonas semiáridas, de semillas mejoradas y clones elite de E. globulus mejor adaptados a la sequía y con mejor rendimiento que aquellos usados comúnmente, así como transformar algunos de esos clones incorporándole genes específicos capaces de expresar una tolerancia aún mayor al estrés hídrico. Al respecto, en este documento se resumen los resultados de una de las estrategias empleadas en el proyecto, particularmente la experiencia con el uso de polinización controlada de madres de un huerto semillero clonal con polen de árboles plus seleccionados por su resistencia a sequía, con el objeto de generar semilla de híbridos intraespecíficos que combinen la superioridad genética de los árboles del huerto con la tolerancia a la sequía de los árboles seleccionados en la raza local.

\section{OBJETIVOS}

Generar híbridos intraespecíficos que combinen la superioridad genética en volumen de árboles de un huerto semillero clonal con la resistencia a la sequía de árboles selectos en condiciones de estrés hídrico. 
Estimar los parámetros genéticos de individuos de E. globulus de 24 familias de polinización controlada y 61 de polinización abierta, de entre 5 y 6 años d edad, y de controles de semilla comercial de $E$. globulus y especies tolerantes a sequía como $E$. camaldulensis y $E$. cladocalyx.

Desarrollar estrategias de mejora para incrementar la resistencia a sequía de E. globulus en el secano de la región del Biobío.

\section{MATERIAL Y METODO}

\section{Selección de Árboles Plus de E. globulus Tolerantes a Sequía: Población de Raza Local}

Se seleccionó un conjunto de 107 árboles plus que exhibían un desempeño sobresaliente de volumen, forma y sanidad en 12.000 hectáreas de plantaciones establecidas en sitios con altas restricciones hídricas entre las región de Coquimbo y el secano de la región del Biobío (Cuadro N¹).

\section{Cuadro $\mathrm{N}^{\circ} 1$}

CRITERIOS PARA SELECCIÓN DE ÁRBOLES PLUS EN PLANTACIONES DE RAZA LOCAL

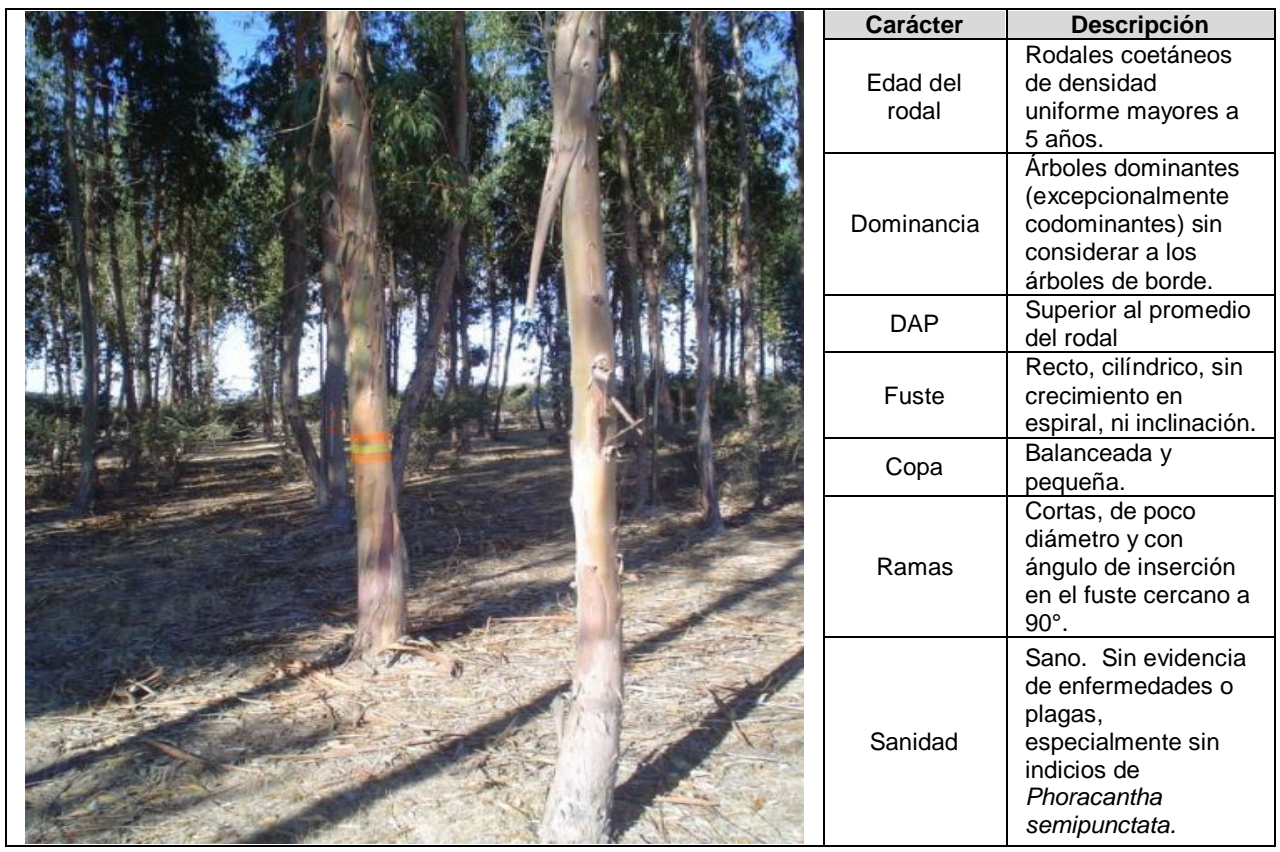

\section{Colecta de Yemas Florales y Procesamiento de Polen}

Desde los árboles selectos se extrajeron secciones de ramas de aproximadamente 40 $\mathrm{cm}$ de longitud y que portaban yemas en estado de ántesis, es decir en la fase de desarrollo floral inmediatamente anterior al desprendimiento del opérculo.

Las ramas envueltas en papel húmedo se depositaron en cajas térmicas con hielo y se 
despacharon al laboratorio de INFOR en Concepción, donde fueron depositas en jarros con agua, etiquetados con el código de cada clon en espera de la apertura de las flores.

Una vez iniciada la apertura de las flores, se les extrajo sus anillos estaminales con un bisturí de punta curva. El material extraído fue secado por 24 a 48 horas en recipientes desecadores con silicagel a temperatura de 25 a $30^{\circ} \mathrm{C}$. Posteriormente, los estambres ya secos fueron agitados sobre láminas de papel de aluminio para desprender el polen. El polen obtenido fue depositado en tubos eppendorf, etiquetados con el código del clon y fecha de colecta, los tubos fueron dispuestos dentro de un frasco con sílicagel, el que fue cerrado y almacenado en freezer hasta el momento de su análisis y utilización en polinización controlada.

Las pruebas de germinación del polen se efectuaron en un medio líquido consistente en una solución al $30 \%$ de sacarosa en agua destilada, complementada con $150 \mathrm{mg} / \mathrm{L}$ de ácido bórico y esterilizada en autoclave por 10 minutos a 70 psi. Este medio de germinación se almacenó congelado hasta el momento de utilizarlo, ocasión en que se descongeló en un horno de microondas. Para analizar la viabilidad del polen se aplicó una pequeña cantidad del mismo en un frasco snap con $2 \mathrm{ml}$ del medio de cultivo, etiquetado con el código del clon, y se incubó por 36 horas a $25^{\circ} \mathrm{C}$. Posteriormente se traspasó una gota del medio con el polen a un portaobjeto y se observó en microscopio óptico con aumento de 100X para determinar el porcentaje de granos de polen germinados. Esto último se determinó por conteo de aquellos granos que exhibían la emisión de tubos polínicos con un largo mayor al diámetro del polen (Figura $N^{\circ} 1$ ).

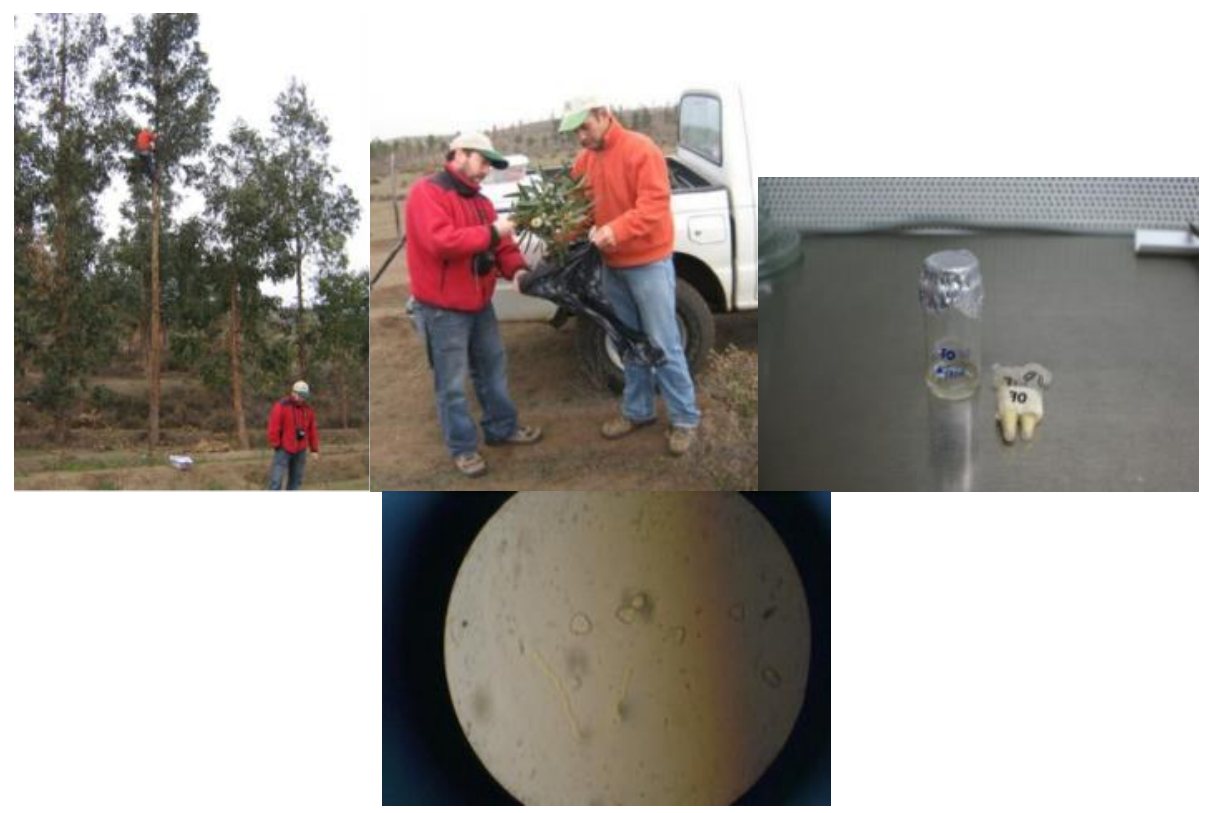

Figura $\mathrm{N}^{\circ} 1$

COLECTA DE YEMAS FLORALES Y PROCESAMIENTO DE POLEN

A nivel de clones la viabilidad in vitro del polen fluctuó entre 0 y $23 \%$, con un promedio ponderado por clon de 7,3\%. Al respecto, se considera que el polen de las especies de Eucalyptus es apropiado para realizar cruzamientos cuando su viabilidad es superior al $5 \%$.

Por lo mismo, aun cuando hubo clones cuyo polen germinó en menos del $5 \%$, el promedio general de todo el material colectado $(7,3 \%)$ excede el valor mínimo aceptado, de modo 
que para efectos de realizar la polinización controlada es posible usar la mezcla total del polen de todos los clones, o alternativamente usar sólo aquel de mayor germinación, tal como se hizo en las actividades del proyecto que se describen más adelante.

Por otra parte, considerando que para polinizar cada flor se requiere aproximadamente $0,0007 \mathrm{~g}$ de polen, se contó con un stock suficiente para polinizar aproximadamente 10.000 flores, lo que resultó más que suficiente para las dos temporadas de cruzamientos contempladas en el proyecto.

\section{Generación de Híbridos Intraespecíficos}

Las labores de polinización controlada se efectuaron en un huerto semillero clonal perteneciente al vivero Agromen, localizado en la localidad de Confluencia (Chillán, región del Biobío).

El huerto cuenta con una superficie de 1,5 ha, donde se distribuyen rametos de 30 clones del programa de mejora genética de INFOR, plantados en bloques al azar, con un espaciamiento de $4 \times 4$ metros.

Como madres se seleccionaron clones del huerto de acuerdo a su ranking genético, disponibilidad de flores y facilidad de acceso a las mismas con escalas de 3 metros. Los cruzamientos se realizaron utilizando la técnica de polinización en una visita (Figura $N^{\circ} 2$ ), la cual se basas en los siguientes principios:

No requiere efectuarse en el momento en que el estigma está maduro y receptivo al grano de polen.

Asegura el éxito del trabajo de emasculación y polinización, y consecuentemente la fertilización de los óvulos.

La polinización y la aislación de las flores polinizadas se realizan en una sola operación.

Se reduce el costo de polinización y se incrementa el número de semillas por flor polinizada.

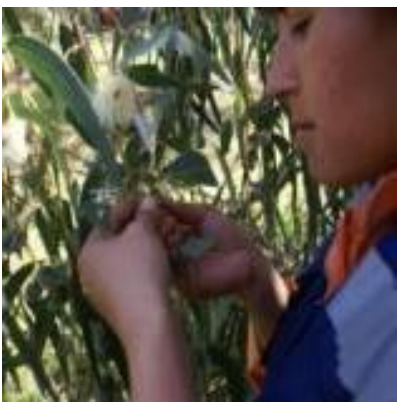

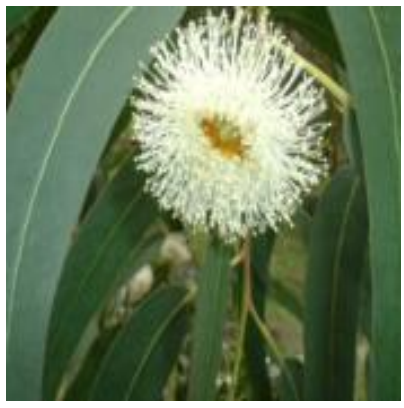

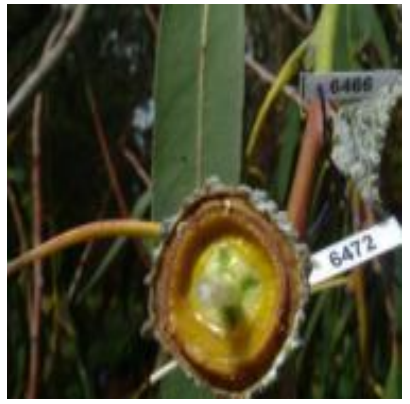

Figura $\mathrm{N}^{\circ} 2$

CRUZAMIENTOS DE E. globulus USANDO TÉCNICA DE POLINIZACIÓN EN UNA VISITA

Cada flor polinizada fue etiquetada con un código que hace referencia al número de la cruza y la identificación de los árboles padre y madre involucrados en la misma. Posteriormente, una vez que los frutos originados en las flores polinizadas alcanzaron su madurez, estos fueron colectados en bolsas de papel identificadas con el código del cruzamiento y transportadas a laboratorio. 
Las cápsulas fueron secadas en horno a $25^{\circ} \mathrm{C}$ y dispuestas en discos petri para la extracción y conteo de sus semillas. Las semillas fueron procesadas manualmente, separándolas de la paráfisis, en base a su color más oscuro, y luego almacenadas en cámara de frio a $4^{\circ} \mathrm{C}$ hasta el momento de su siembra en vivero.

\section{Producción en Vivero de Familias de Polinización Abierta y Controlada}

La producción de plantas, tanto de las familias de polinización abierta como controlada, fue realizada en el vivero Agromen (Figura $N^{\circ} 3$ ), planificando una producción esperada de 200 plantas por familia, para efectos de disponer de 50 unidades de cada familia en cada ensayo y contar con un excedente para las filas de aislación o borde de los mismos.
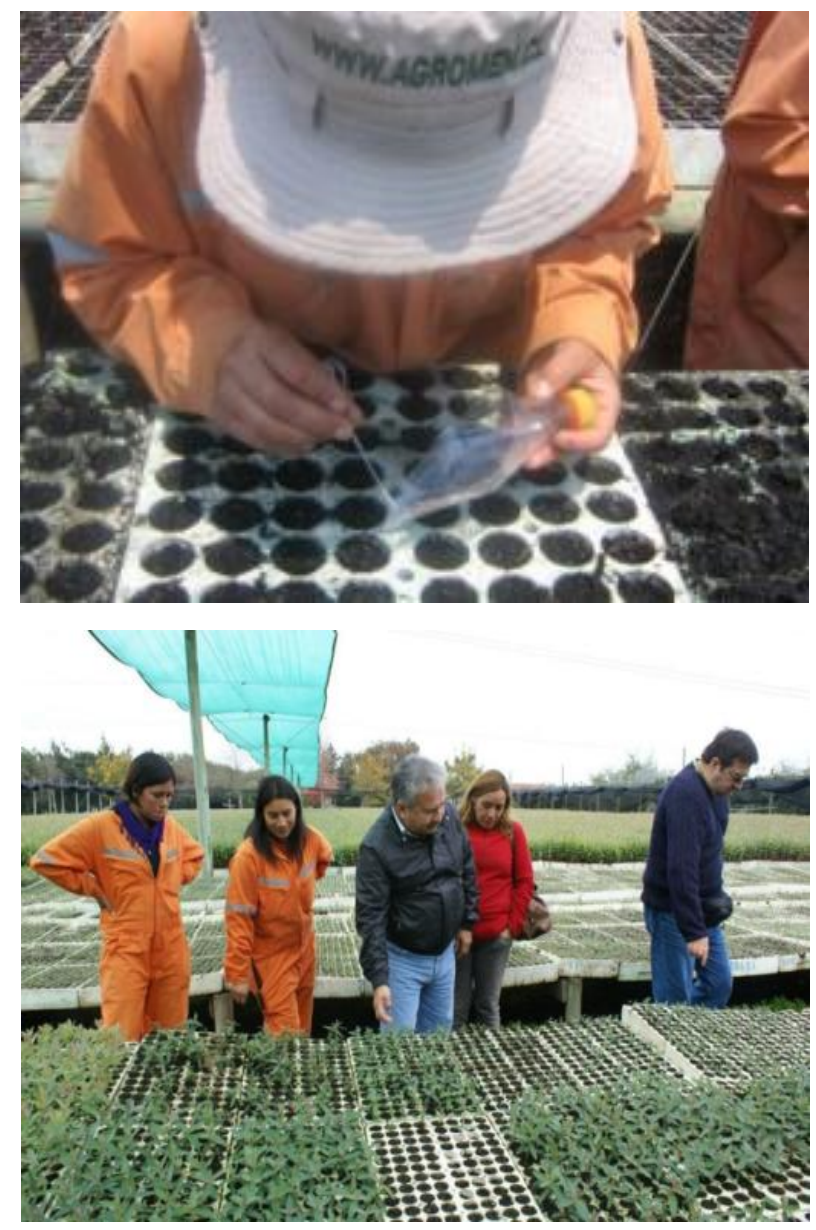

Figura $\mathbf{N}^{\circ} 3$

PRODUCIÓN DE PLANTAS DE E. globulus EN VIVERO 
La producción de plantas se efectuó en bandejas de poliestireno expandido con cavidades individuales de $130 \mathrm{cc}$, utilizando como sustrato corteza compostada de pino.

El material genético para los ensayos de polinización abierta consideró un total de 72 progenies correspondientes a:

56 Progenies de árboles plus seleccionados en condiciones de aridez (selecciones raza local)

13 Progenies australianas de árboles de alta productividad del programa de mejora de INFOR, representados en un huerto semillero clonal (madres australianas en HSC)

3 Lotes de plantas de comparación: Uno correspondiente a plantas de semilla comercial de E. globulus, y otros dos correspondientes a plantas de $E$. camaldulensis y $E$. cladocalyx, especies reconocidas por su adaptación a condiciones de aridez (controles).

El material genético para los ensayos de polinización controlada consideró a las 37 progenies que se detallan a continuación:

30 Progenies híbridas intraespecíficas que combinan la superioridad genética de árboles de un huerto semillero clonal con la tolerancia a sequía de árboles de raza local seleccionados en condiciones de aridez en plantaciones comerciales.

7 Progenies de polinización abierta de las mismas madres representadas en el huerto clonal.

\section{Ensayos de Progenies}

Los ensayos fueron plantados en áreas representativas del interior de la región del Biobío, donde la especie E. globulus enfrenta restricciones hídricas que restringen su tasa de crecimiento.

Los ensayos de polinización abierta se plantaron en La Posada y San Vicente, mientras que el ensayo de polinización controlada fue establecido en El Mirador.

Los tres sitios corresponden al valle interior, entre el Océano Pacífico y la Cordillera de los Andes, en la zona denominada como secano interior donde la productividad de E. globulus es considerablemente más baja que en la costa, llegando solo a $10-15 \mathrm{~m}^{3} / \mathrm{ha} / \mathrm{año}$.

En la Figura $\mathrm{N}^{\circ} 4$ se presenta la ubicación de los ensayos e información relativa los montos anuales de precipitación registrados en la zona de emplazamiento de los mismos.

Al comparar los requerimientos climáticos de E. globulus mencionados por Booth y Pryor (1991) con los registros de precipitación de la Figura $N^{\circ} 4$, se observa que el monto de precipitación anual es coincidente con el requerimiento de la especie.

No obstante, el número de meses secos, la temperatura media anual y la temperatura del mes más cálido, son mayores al rango de requerimientos de E. globulus.

Los ensayos fueron plantados en septiembre de 2009 y septiembre de 2010 , considerando silvicultura intensiva de establecimiento, la que incluyó preparación de suelo con subsolador, control químico pre y post emergencia de malezas, además de fertilización individual de las plantas.

Los espaciamientos de plantación utilizados fueron de $3 \times 2,5 \mathrm{~m}$ en La Posada y El Mirador, y de $3 \times 3 \mathrm{~m}$ en San Vicente. En el Cuadro $\mathrm{N}^{\circ} 2$ se resumen los antecedentes de los tres ensayos considerados. 




Figura $\mathrm{N}^{\circ} 4$

UBICACIÓN DE LOS ENSAYOSY ANTECEDENTES DE PRECIPITACIÓN ANUAL 
Cuadro $\mathrm{N}^{\circ} 2$

ANTECEDENTES DE LOS ENSAYOS DE PROGENIES

\begin{tabular}{|c|c|c|c|}
\hline \multirow{2}{*}{ Característica } & \multicolumn{3}{|c|}{ Ensayos } \\
\hline & La Posada & San Vicente & El Mirador \\
\hline Plantación & Septiembre, 2010 & Septiembre, 2009 & Septiembre, 2010 \\
\hline Espaciamiento & $3,0 \times 2,5 \mathrm{~m}$ & $3,0 \times 3,0 \mathrm{~m}$ & $3,0 \times 2,5 \mathrm{~m}$ \\
\hline Superficie & 0,9 ha & 1,36 ha & 0,65 ha \\
\hline Diseño & $\begin{array}{l}6 \text { bloques completos al azar } \\
\text { Parcelas lineales de } 4 \text { árboles } \\
\text { por progenie } \\
50 \text { progenies por bloque. }\end{array}$ & $\begin{array}{l}6 \text { bloques completos al azar } \\
\text { Parcelas lineales de } 4 \text { árboles } \\
\text { por progenie } \\
63 \text { progenies por bloque. }\end{array}$ & $\begin{array}{l}6 \text { bloques completos al azar } \\
\text { Parcelas lineales de } 4 \text { árboles } \\
\text { por progenie } \\
36 \text { progenies por bloque. }\end{array}$ \\
\hline Aislación & $\begin{array}{l}\text { Una hilera de } E \text {. } \\
\text { camaldulensis y plantación } \\
\text { comercial de E. globulus. }\end{array}$ & $\begin{array}{l}\text { Plantación comercial de } E \text {. } \\
\text { globulus }\end{array}$ & $\begin{array}{l}\text { Plantación comercial de } E \text {. } \\
\text { globulus }\end{array}$ \\
\hline $\begin{array}{l}\text { Preparación } \\
\text { suelo }\end{array}$ & $\begin{array}{l}\text { Casillas de } 30 \times 30 \times 30 \mathrm{~cm} \\
\text { confeccionadas con pala } \\
\text { plantadora. }\end{array}$ & Subsolado y mullido & Subsolado y mullido \\
\hline $\begin{array}{l}\text { Silvicultura de } \\
\text { establecimiento }\end{array}$ & \multicolumn{3}{|c|}{ Control químico pre y post emergente de malezas y fertilización. } \\
\hline Suelo* & $\begin{array}{l}\text { Suelos residuales, formados a } \\
\text { partir de rocas graníticas, bien } \\
\text { evolucionados, de texturas } \\
\text { arcillosas en todo el perfil. } \\
\text { Descansa sobre un substrato } \\
\text { constituido por roca granítica } \\
\text { muy meteorizada y rica en } \\
\text { cuarzo y feldespatos }\end{array}$ & $\begin{array}{l}\text { Suelo moderadamente } \\
\text { profundo a profundo, bien } \\
\text { evolucionado, derivado de } \\
\text { sedimentos aluviales } \\
\text { mezclados con un aporte de } \\
\text { cenizas variables. } \\
\text { Descansa sobre substrato de } \\
\text { diversos orígenes }\end{array}$ & $\begin{array}{l}\text { Suelo derivado de rocas } \\
\text { metamórficas y graníticas, } \\
\text { presenta textura franco- } \\
\text { arcillosa y un pH del orden de } \\
6,0 \text {. } \\
\text { Niveles bajos de nutrientes y } \\
\text { materia orgánica y baja } \\
\text { capacidad de retención de } \\
\text { agua. }\end{array}$ \\
\hline Clima & $\begin{array}{l}\mathrm{N}^{\circ} \text { de meses secos: } 4 \\
\mathrm{~T}^{\circ} \text { máxima: } 38^{\circ} \mathrm{C} \\
\mathrm{T}^{\circ} \text { media anual: } 13^{\circ} \mathrm{C} \\
\mathrm{T}^{\circ} \text { mínima absoluta: }-8^{\circ} \mathrm{C}\end{array}$ & $\begin{array}{l}\mathrm{N}^{\circ} \text { de meses secos: } 4 \\
\mathrm{~T}^{\circ} \text { máxima: } 38^{\circ} \mathrm{C} \\
\mathrm{T}^{\circ} \text { media anual: } 13^{\circ} \mathrm{C} \\
\mathrm{T}^{\circ} \text { mínima absoluta: }-8^{\circ} \mathrm{C}\end{array}$ & $\begin{array}{l}\mathrm{N}^{\circ} \text { de meses secos: } 4 \\
\mathrm{~T}^{\circ} \text { máxima: } 34^{\circ} \mathrm{C} \\
\mathrm{T}^{\circ} \text { media anual: } 15^{\circ} \mathrm{C} \\
\mathrm{T}^{\circ} \text { mínima absoluta: }-6^{\circ} \mathrm{C}\end{array}$ \\
\hline
\end{tabular}

(*Fuente: Santis, 2005)

\section{Medición y Evaluación Genética de los Ensayos de Progenies}

En septiembre y octubre de 2015 se realizó la medición de los ensayos, registrándose los valores de altura y díametro a la altura del pecho de cada uno de sus árboles. Para este efecto se utilizó vertex, huincha diamétrica y formularios de terreno en los cuales estaban registrados los valores de la medición anterior, esto con el objeto de validar y evitar errores en la captura de los datos.

Adicionalmente, durante la medición se tomo información de algunas variables subjetivas, tales como rectitud de fuste, hábito de crecimiento y sanidad, particualemente ataque de Phoracantha semipunctata y Gonipterus scutellatus.

Para el análisis genético de los ensayos de progenie se utilizó la última medición de altura y diámetro en cada sitio. Debido a que la diferencia en edad entre los sitios es pequeña, se optó por no efectuar correcciones por cantidad de mediciones del tipo de Wei y Borralho (1996).

Los registros de la base de datos fueron ordenados por sitio y contenían información para ambas variables. Fueron considerados como missing value los árboles muertos y de relleno, con daños o con más de un fuste. Se utilizó un modelo de árbol individual o animal model (Borralho, 1995): 


$$
y=X b+Z_{u} u+e
$$

Donde:

$\boldsymbol{y}$ es el vector de las observaciones para altura y diámetro en todos los sitios, $\boldsymbol{b}$ es el vector de parámetros para los efectos fijos (sitio y bloque),

$\boldsymbol{u}$ es el vector de valores genéticos para las dos variables dentro de cada sitio, $\boldsymbol{e}$ es el vector de residuos aleatorios.

$\boldsymbol{X}, \boldsymbol{Z}_{u}$ y representan respectivamente la matrices de diseño para los efectos fijos y efectos aleatorio o valores genéticos.

El análisis de datos fue desarrollado en tres etapas. Primero un análisis separado o univariado para cada variable en cada sitio, es decir para altura $(\mathrm{m})$ y diámetro, incluyendo bloque, sitio, valor genético del árbol y error para obtener valores iniciales para los componentes de varianza. La segunda etapa involucró un análisis bivariado altura $(\mathrm{m})$ y DAP $(\mathrm{cm})$ y finalmente un análisis multivariado y multisitio (el modelo completo) para obtener los componentes de varianza y la mejor predicción lineal insesgada (BLUP=Best Linear Unbiased Predictor) de los valores genéticos usando las ecuaciones de modelos lineales mixtos de Henderson (1984).

Los componentes de varianza fueron estimados usando Máxima Verosimilitud Restringida (REML, Patterson y Thompson 1971). Para esto se utilizó el programa ASREML (Gilmour et al., 1995, Gilmour et al., 1997) que mediante el uso de un algoritmo iterativo de información promedio y técnicas de sparse matrices reduce en forma considerable los tiempos de análisis y requerimientos computacionales. Además de los componentes de varianza, se estimaron las heredabilidades $\left(h^{2}\right)$ y correlaciones genéticas $\left(r_{g}\right)$ de acuerdo a las siguientes ecuaciones:

$$
\begin{gathered}
h^{2}=\frac{\sigma_{a}{ }^{2}}{\sigma_{a}{ }^{2}+\sigma_{e}{ }^{2}} \\
r_{g}=\frac{\sigma_{a_{1} a_{2}}}{\sigma_{a_{1}} \sigma_{a_{2}}}
\end{gathered}
$$

Los errores estándares de los parámetros genéticos fueron obtenidos directamente con ASREML, basado en los términos de la inversa de la matriz de coeficientes de las ecuaciones de modelos lineales (Gilmour et al., 1997).

Los valores genéticos de Altura y DAP de cada árbol individual se utilizaron para confeccionar un índice correspondiente al promedio de ambas variables ponderadas por sus respectivas heredabilidades.

El ordenamiento de mayor a menor de este índice dio origen a los rankings genéticos, los cuales se elaboraron para cada ensayo y se diferenciaron a nivel de árboles individuales, de familias y de grupos. En este último caso los grupos estuvieron conformados por el origen del material (madres australianas en huerto semillero clonal, selecciones de raza local en secano y controles).

En el caso del ensayo de polinización controlada, además del ranking familiar por cruza se agregan dos rankings adicionales, los que corresponden a los ordenamientos jerarquizados por madre y por padre. La utilidad de los rankings mencionados es apoyar al mejorador en el proceso de la selección hacia adelante, para la siguiente generación del programa de mejora genética. 


\section{RESULTADOS}

\section{Parámetros Genéticos y Correlaciones}

El Cuadro $N^{\circ} 3$ muestra la heredabilidad en sentido estricto, y su respectivo error estándar, estimada para las variables altura y DAP en cada uno de los tres ensayos evaluados.

La estimación de heredabilidad corresponde a la obtenida con un coeficiente de parentesco $r=0,25$ (medios hermanos), usado solo para efectos de comparación.

\section{Cuadro $\mathrm{N}^{\circ} 3$ \\ HEREDABILIDADES $\left(h^{2}\right)$ Y SUS RESPECTIVOS ERRORES ESTÁNDAR (EE) PARA ALTURA Y DAP EN CADA ENSAYO}

\begin{tabular}{|l|c|c|}
\hline \multirow{2}{*}{ Ensayo } & \multicolumn{2}{|c|}{$\mathbf{h}^{2} \pm \mathbf{E E}$} \\
\cline { 2 - 3 } & Altura & DAP \\
\hline La Posada & $0.4323 \pm 0.0247$ & $0.3346 \pm 0.0291$ \\
\hline San Vicente & $0.5503 \pm 0.0173$ & $0.4919 \pm 0.0196$ \\
\hline El Mirador & $0.3624 \pm 0.0424$ & $0.2542 \pm 0.0496$ \\
\hline
\end{tabular}

Las correlaciones genética aditiva y fenotípica entre las variables Altura y DAP de cada sitio, con sus respectivos errores estándar, se presentan en el Cuadro $\mathrm{N}^{\circ} 4$. Estos valores fluctúan entre - 1 y 1 , donde cero indica que no existe correlación.

\section{Cuadro $\mathrm{N}^{\circ} 4$}

CORRELACIONES ENTRE ALTURA Y DAP EN CADA ENSAYO

\begin{tabular}{|l|c|c|}
\hline \multirow{2}{*}{ Ensayo y variables } & \multicolumn{2}{|c|}{ Correlaciones } \\
\cline { 2 - 3 } & Genética \pm EE & Fenotípica \pm EE \\
\hline La Posada, Altura-DAP & $0.9261 \pm 0.0237$ & $0.8017 \pm 0.0110$ \\
\hline San Vicente, Altura-DAP & $0.8767 \pm 0.0178$ & $0.7720 \pm 0.0110$ \\
\hline El Mirador, Altura-DAP & $0.9394 \pm 0.0707$ & $0.6837 \pm 0.0250$ \\
\hline
\end{tabular}

Las correlaciones genéticas para una misma variable entre pares de sitios (ensayos) indican el grado de homogeneidad en el comportamiento de los rankings individuales entre esos sitios.

Así, una alta correlación es indicativa de ausencia de interacción genotipo ambiente, señalando que los rankings son estables o similares en ambos sitios, es decir que los mejores árboles en un ensayo, son también los mejores en el otro.

Por el contrario un bajo nivel de correlación estaría indicando que los rankings de cada ensayo son diferentes y que existe efecto de la interacción genotipo-ambiente.

Al respecto, en el Cuadro $\mathrm{N}^{\circ} 5$ se indican las correlaciones entre pares de sitios para la variable altura, con su respectivo error estándar.

Lo mismo ocurre en el Cuadro $\mathrm{N}^{\circ} 6$ para la variable DAP. 
Cuadro $\mathrm{N}^{\circ} 5$

CORRELACIÓN GENÉTICA ADITIVA Y ERROR ESTÁNDAR PARA ALTURA ENTRE ENSAYOS

\begin{tabular}{|l|c|c|c|}
\cline { 2 - 4 } \multicolumn{1}{c|}{} & La Posada & San Vicente & El Mirador \\
\hline La Posada & - & & \\
\hline San Vicente & $0.0044 \pm 0.0003$ & - & \\
\hline EI Mirador & $0.0057 \pm 0.0006$ & $0.0041 \pm 0.0004$ & - \\
\hline
\end{tabular}

Cuadro $\mathrm{N}^{\circ} 6$

CORRELACIÓN GENÉTICA ADITIVA Y ERROR ESTÁNDAR PARA DAP ENTRE ENSAYOS

\begin{tabular}{|l|c|c|c|}
\cline { 2 - 4 } \multicolumn{1}{c|}{} & La Posada & San Vicente & El Mirador \\
\hline La Posada & - & & \\
\hline San Vicente & $0.0030 \pm 0.0002$ & - & \\
\hline El Mirador & $0.0045 \pm 0.0007$ & $0.0032 \pm 0.0004$ & - \\
\hline
\end{tabular}

\section{Análisis Genético Ensayo La Posada (polinización abierta)}

El ranking de árboles individuales del ensayo La Posada (Apéndice $N^{\circ} 1$ ) muestra que en sus primeras posiciones existe un claro predominio de los descendientes (hijos) de los árboles de raza local seleccionados en condiciones de secano, en desmedro de los individuos originados a partir de las madres australianas representadas en el huerto semillero clonal. Estos últimos presentan una participación muy marginal en la cabecera del ranking, recién en la posición 25 figura un hijo de la madre australiana $\mathrm{N}^{\circ} 33$ (Jeerelang, Norte de Victoria).

El individuo de mejor desempeño es hijo de una selección de raza local, particularmente de la $N^{\circ} 57$ efectuada en Cauquenes, la cual se destaca por presentar a varios de sus hijos en la cabecera del ranking.

El ranking a nivel familiar (Apéndice $\mathrm{N}^{\circ} 2$ ) confirma el buen desempeño de la familia $\mathrm{N}^{\circ}$ 57 y de otras selecciones de raza local. En general las familias derivadas de madres australianas, en este ensayo, presentan un desempeño inferior al de las selecciones de raza local. Por su parte, los grupos testigos formados por material de E. camaldulensis y E. cladocalyx presentan rendimientos claramente inferiores a E. globulus.

Agrupando el material ensayado a nivel de grandes grupos (Cuadro $\mathrm{N}^{\circ} 7$ ) se hace evidente el mejor desempeño de las selecciones de raza local en relación a las madres australianas del huerto semillero.

Cuadro $\mathrm{N}^{\circ} 7$

RANKING POR GRUPO DE MATERIAL GENÉTICO EN ENSAYO DE POLINIZACIÓN ABIERTA LA POSADA

\begin{tabular}{|l|c|c|}
\hline \multicolumn{1}{|c|}{ Grupo de Material Genético } & Índice & Ranking \\
\hline $\begin{array}{l}\text { Progenies de polinización abierta de selecciones de } \\
\text { raza local }\end{array}$ & 0,083 & 1 \\
\hline $\begin{array}{l}\text { Progenies de polinización abierta de madres } \\
\text { australianas en huerto semillero clonal }\end{array}$ & $-0,070$ & 2 \\
\hline Control E. cladocalyx & $-1,133$ & 3 \\
\hline Control E. camaldulensis & $-2,008$ & 4 \\
\hline
\end{tabular}


En promedio solo el material de secano exhibe valores de mejora promedios superiores a cero, mientras que los tres grupos restantes presentan valores negativos.

\section{Análisis Genético Ensayo San Vicente (polinización abierta)}

El ranking de árboles individuales (Apéndice $\mathrm{N}^{\circ} 3$ ) y el familiar (Apéndice $\mathrm{N}^{\circ} 4$ ) denotan un comportamiento distinto al observado en el ensayo La Posada.

En San Vicente se observa una mayor participación de progenies de madres australianas en la parte alta del ranking. De hecho, la familia de mejor desempeño en este ensayo corresponde a la madre australiana $\mathrm{N}^{\circ} 7$.

Lo mismo ocurre en el ranking de árboles individuales, donde el mejor individuo proviene de una madre de ese origen, y donde se observa una alta participación de este material en la cabecera del ranking.

La situación descrita se confirma y hace más evidente en el ordenamiento a nivel de grupos (Cuadro $\mathrm{N}^{\circ} 8$ ), donde se indica que el mejor desempeño corresponde al material australiano, seguido de la raza local, mientras que los controles E. camaldulensis y E. cladocalyx siguen siendo los grupo de menor desempeño

\section{Cuadro $N^{\circ} 8$ \\ RANKING POR GRUPO DE MATERIAL GENÉTICO EN ENSAYO DE POLINIZACIÓN ABIERTA SAN VICENTE}

\begin{tabular}{|l|c|c|}
\hline \multicolumn{1}{|c|}{ Grupo de Material Genético } & Índice & Ranking \\
\hline $\begin{array}{l}\text { Progenies de polinización abierta de madres } \\
\text { australianas en huerto semillero clonal }\end{array}$ & 0,636 & $\mathbf{1}$ \\
\hline $\begin{array}{l}\text { Progenies de polinización abierta de selecciones } \\
\text { de raza local }\end{array}$ & $-0,046$ & $\mathbf{2}$ \\
\hline Control E. cladocalyx & $-2,533$ & $\mathbf{3}$ \\
\hline Control E. camaldulensis & $-2,590$ & $\mathbf{4}$ \\
\hline
\end{tabular}

\section{Análisis Genético Ensayo El Mirador (polinización controlada)}

En este ensayo resulta destacable el comportamiento superior exhibido por los híbridos intraespecíficos derivados de cruzamientos controlados efectuados con polen de árboles padre de raza local, sobre madres australianas del huerto semillero.

El lote control de E. globulus (semilla comercial) tiene un desempeño intermedio entre todo el material ensayado, resultando en promedio mejor que el material de polinización abierta producido en el huerto.

Al igual que en los ensayos anteriores, el control E. camaldulensis muestra el desempeño más bajo, mientras que el $E$. cladocalyx no fue capaz de sobrevivir en las condiciones de este ensayo (Cuadro $\mathrm{N}^{\circ}$ 9).

Los primeros 6 árboles del ranking individual (Apéndices $\mathrm{N}^{\circ} 5$ ) son hijos de la cruza de la madre australiana $\mathrm{N}^{\circ} 40$ con el padre de raza local $\mathrm{N}^{\circ} 72$. Consistentemente, esta misma cruza es la que presenta la mejor aptitud combinatoria específica, situación que se confirma en el ranking en función de las cruzas (Apéndice $\mathrm{N}^{\circ} 6$ ).

Adicionalmente, estos mismos árboles madre y padre exhiben también la mejor aptitud combinatoria general, situación que se corrobora en los rankings ordenados por madre (Apéndice 


\section{Cuadro $N^{\circ} 9$ \\ RANKING POR GRUPO DE MATERIAL GENÉTICO EN ENSAYO DE POLINIZACIÓN CONTROLADA EL MIRADOR}

\begin{tabular}{|l|c|c|}
\hline \multicolumn{1}{|c|}{ Grupo de Material Genético } & Índice & Ranking \\
\hline $\begin{array}{l}\text { Progenies de polinización controlada de madres } \\
\text { australianas con polen de selecciones de raza local }\end{array}$ & 0,287 & $\mathbf{1}$ \\
\hline Control E. globulus & 0,287 & $\mathbf{2}$ \\
\hline $\begin{array}{l}\text { Progenies de polinización abierta de madres australianas } \\
\text { en huerto semillero clonal }\end{array}$ & $-0,076$ & $\mathbf{3}$ \\
\hline Control E. camaldulensis & $-1,470$ & $\mathbf{4}$ \\
\hline
\end{tabular}

\section{DISCUSION Y CONCLUSIONES}

De acuerdo con Potts and Dutkowsky (2012) existen diferencias significativas en la susceptibilidad al daño por sequía en las subrazas de $E$. globulus resultando en una amplia variación evidente a escala regional o de clines. La mayor tolerancia a sequía se asocia con la resistencia al cerambícido Phoracantha semipunctata. Los autores concluyen que los origenes de E. globulus más susceptibles a la sequía corresponden a King Island y la costa oeste de Tasmania, mientras que los más tolerantes son los de Jeerelang y el norte de Tasmania.

La supervivencia inicial de las pruebas de progenie de polinización abierta fue de $92 \%$ gracias a la intensiva silvicultura de establecimiento. El ensayo de polinización controlada evidenció una supervivencia menor, $63 \%$ a los 20 meses, debido a daño por animales, por lo mismo el bloque que concentró este problema fue excluido del análisis genético.

Los altos valores de la heredabilidad estimada para altura y DAP ponen de manifiesto la excelente calidad de los ensayos y generan expectativas de aumentar la ganancia genética mediante selección a nivel individual y de familias para el material de origen australiano y para las selecciones de raza local. Estos resultados han sido obtenidos tras un largo periodo de sequía que se ha extendido por 14 años en la zona donde se ubican los ensayos, de modo que es posible albergar grandes expectativas de ganancia genética en años con más precipitación.

Las corelaciones genéticas entre sitios se muestran cercanas a cero, indicando una alta interacción del material genético con las condiciones de sitio de los ensayos. Esta obedece principalmente a las diferencias en características del suelo y variación microclimática entre las áreas de Chillán y San Carlos.

Las evidentes diferencias entre los rankings de San Vicente y La Posada dan cuenta de la incidencia de la interacción genotipo ambiente y se pueden explicar en función de diferencias ambientales entre los sitios de ensayo, las cuales favorecen de forma preferencial el desempeño del material de secano en el sitio del primer ensayo. En estas condiciones es recomendable el uso de rankings y selecciones específicas para cada sitio, más que de un ranking conjunto para evaluarlos a ambos simultáneamente.

En el ensayo El Mirador los híbridos intraespecíficos que combinan atributos de las madres australianas del huerto semillero, con padres tolerantes a sequía de la raza local, exhiben mejor desarrollo que las progenies de polinización abierta de las madres australianas. La mejor combinación específica corresponde a la madre australiana $N^{\circ} 40$ (Suroeste de Apollo Bay en Victoria), con el padre de raza local $N^{\circ} 72$ (árbol seleccionado en Ovalle, región de Coquimbo) 


\section{REFERENCIAS}

Booth, T. y Pryor L. 1991. Climatic requirements of some commercially important Eucalypt species Forest Ecology and Management 43(1991)

Borralho, N. 1995. The impact of individual tree mixed model methods (BLUP) in tree breeding strategies. En: Eucalyptus Plantation: Improving Fibre Yield and Quality (Eds. B. Potts, N. Borralho, J. Reid, R. Cromer, W. Tibbits y C. Raymond). pp. 141-145. Proc. CRC-IUFRO. Conf., Hobart, 19-24 Feb. CRC for Temperate Hardwood Forestry, Hobart (Australia).

Cauvin, B. 1988. Pistil treatments for improved fertility in hybridization of $E$. gunnii (Hook). In sexual reproduction in Higher plants: proceedings of the $10^{\text {th }}$ International Symposium on the sexual reproduction of the high plants, $30^{\text {th }}$ May to $4^{\text {th }}$ June 1988. University of Siena, Siena, Italy. (Ed. PG M. Cresti, E. Pacini) pp $321-325$ (SpirngerVerlag, Berlin).

Dutkowsky, G. 1995 . Genetic varation in drought susceptibility of Eucalyptus globulus ssp globulus in plantations in Western Australia. In : Potts BM, Borralho NMG, Reid JB, Cromer RN, Tibbits WN, Raymond CA (eds) Eucalypt plantations : improving the fibre yield and quality. CRC for temperate Hardwood Forestry, Hobart, Tasmania, pp 199-203

Dutkowski, G. and Potts, B. 1999. Geographical patterns of genetic variation in Eucalyptus globulus ssp. globulus and a revised racial classification. Aust J Bot 46:237-263

Dutkowski G.; Potts, B.; Borralho, N. 1997. Revised racial classification of Eucalyptus globulus ssp. globulus and the importance of including race in analysis of progeny trials. IUFRO Conference on Silviculture and Improvement of Eucalypts. EMBRAPA, Colombo, Salvador, Bahia, Brazil, pp 322-329

Espejo, J.; England, N. and Griffin, R. 1995. Results of a crossing program with Eucalyptus nitens and $E$. globulus in Chile. In: Potts, B.; Borralho, N.; Reid, J.; Cromer, R.; Tibbits, W. and Raymond, C. (eds) Eucalypt plantations: improving fibre yield and quality. Proc. CRCTHF-IUFRO Conf, Hobart, 19-24 Feb. CRC for Temperate Hardwood Forestry, Hobart, Tasmania, pp 239-240

Gilmour, A.R.; Thompson, R. and Cullis, B.R. 1995. Average information REML, an efficient algorithm for variance parameter estimation in linear mixed models. Biometrics 51:1440-1450.

Gilmour, A.R.; Thompson, R.; Cullis, B.R. and Welham, S.J. 1997. ASREML user's manual. July 24, 1997. $100 \mathrm{p}$.

Henderson, C.R. 1984. Applications of linear models in animal breeding. University of Guelph Press, Guelph.

Potts, B.M. y Dutkowski, 2012. Genetic variation in the susceptibility of Eucalyptus globulus to drought damage. Tree genetics \& genomes. Springer.

Rojas, P. and Griffin, R., 1997. Fibre Yield Improvement Program (FYIP) of E. globulus Labill in Santa Fe Group. In: Proceedings of the IUFRO Conference on Silviculture and Improvement of Eucalyptus. Salvador, Brasil. Pp 206-211

Rojas, P.; Ramirez de Arellano, P.; Contardo, C.; Balocchi, C. and Potts, B. 2001. Desarrollo de una metodologia para la produccion de semilla hibrida intra e interespecifica en un huerto semillero clonal de Eucalyptus globulus. Developing the Eucalypt of the Future, p 88

Santis, G. 2005. Mapa de reconocimiento de suelos de la región del Biobío (sector Norte). Memoria de Título, Escuela de Agronomía, Facultad de Ciencias Agronómicas, Universidad de Chile.

Toro, M.; Silió, M.; Rodriguez, C.; Soria, F. and Toval, G. 1998. Genetic analysis of survival to drought in Eucalyptus globulus in Spain. Proceedings of the 6th World Congress on Genetics Applied to Livestock Production, Armidale, NSW, Australia, Vol. 27, Pp: 499-502.

Wei, X. y N.M.G. Borralho. 1996. A simple model to describe age trends in heritability in short rotation tree species. En: "Tree improvement for sustainable tropical forestry". (Eds. Dieters, M.J.; A.C. Matheson; D.G. Nikles; C.E. Harwood y S.M. Walkers). pp 178-181. Proc. QFRI-IUFRO Conf., Caloundra, Queensland, Australia. 27 Oct.1 Nov.

Williams, D.; Potts, B. and Black, P. 1999. Testing single visit pollination procedures for Eucalyptus globulus and E. nitens. Australian Forestry 62, 346-352 


\section{APÉNDICE 1 \\ RANKING DE ÁRBOLES INDIVIDUALES DEL ENSAYO LA POSADA \\ (Primer 5\% del ranking)}

\begin{tabular}{|c|c|c|c|c|c|}
\hline Madre & & Bloque & Árbol & Índice & Ranking \\
\hline Selección raza local $N^{\circ}$ & 57 & 4 & 4 & 7,039 & 1 \\
\hline Selección raza local $\mathrm{N}^{\circ}$ & 57 & 4 & 3 & 6,548 & 2 \\
\hline Selección raza local $N^{\circ}$ & 104 & 5 & 1 & 5,656 & 3 \\
\hline Selección raza local $\mathrm{N}^{\circ}$ & 22 & 5 & 4 & 5,483 & 4 \\
\hline Selección raza local $\mathrm{N}^{\circ}$ & 7 & 5 & 3 & 4,804 & 5 \\
\hline Selección raza local $\mathrm{N}^{\circ}$ & 52 & 2 & 3 & 4,560 & 6 \\
\hline Selección raza local $\mathrm{N}^{\circ}$ & 79 & 1 & 1 & 4,527 & 7 \\
\hline Selección raza local $\mathrm{N}^{\circ}$ & 74 & 4 & 4 & 4,507 & 8 \\
\hline Selección raza local $\mathrm{N}^{\circ}$ & 104 & 1 & 1 & 4,481 & 9 \\
\hline Selección raza local $\mathrm{N}^{\circ}$ & 73 & 6 & 1 & 4,461 & 10 \\
\hline Selección raza local $\mathrm{N}^{\circ}$ & 57 & 4 & 2 & 4,457 & 11 \\
\hline Selección raza local $N^{\circ}$ & 7 & 4 & 1 & 4,362 & 12 \\
\hline Selección raza local $N^{\circ}$ & 73 & 6 & 2 & 4,316 & 13 \\
\hline Selección raza local $N^{\circ}$ & 73 & 6 & 4 & 4,302 & 14 \\
\hline Selección raza local $N^{\circ}$ & 104 & 6 & 1 & 4,246 & 15 \\
\hline Selección raza local $\mathrm{N}^{\circ}$ & 104 & 5 & 3 & 4,225 & 16 \\
\hline Selección raza local $N^{\circ}$ & 14 & 2 & 1 & 4,167 & 17 \\
\hline Selección raza local $N^{\circ}$ & 106 & 2 & 4 & 4,060 & 18 \\
\hline Selección raza local $N^{\circ}$ & 57 & 6 & 3 & 4,026 & 19 \\
\hline Selección raza local $\mathrm{N}^{\circ}$ & 79 & 3 & 1 & 3,959 & 20 \\
\hline Selección raza local $\mathrm{N}^{\circ}$ & 57 & 4 & 1 & 3,908 & 21 \\
\hline Selección raza local $\mathrm{N}^{\circ}$ & 57 & 6 & 2 & 3,888 & 22 \\
\hline Selección raza local $\mathrm{N}^{\circ}$ & 57 & 5 & 4 & 3,887 & 23 \\
\hline Selección raza local $N^{\circ}$ & 73 & 6 & 3 & 3,824 & 24 \\
\hline Madre australiana en $\mathrm{HSC} \mathrm{N}^{\circ}$ & 33 & 1 & 2 & 3,804 & 25 \\
\hline Madre australiana en $\mathrm{HSC} \mathrm{N}^{\circ}$ & 1 & 6 & 1 & 3,788 & 26 \\
\hline Madre australiana en $\mathrm{HSC} \mathrm{N}^{\circ}$ & 2 & 4 & 1 & 3,760 & 27 \\
\hline Selección raza local $N^{\circ}$ & 104 & 6 & 2 & 3,697 & 28 \\
\hline Selección raza local $\mathrm{N}^{\circ}$ & 42 & 5 & 4 & 3,676 & 29 \\
\hline Selección raza local $\mathrm{N}^{\circ}$ & 91 & 3 & 1 & 3,662 & 30 \\
\hline Selección raza local $\mathrm{N}^{\circ}$ & 104 & 5 & 2 & 3,660 & 31 \\
\hline Selección raza local $N^{\circ}$ & 16 & 3 & 4 & 3,645 & 32 \\
\hline Selección raza local $\mathrm{N}^{\circ}$ & 104 & 4 & 2 & 3,631 & 33 \\
\hline Selección raza local $\mathrm{N}^{\circ}$ & 27 & 1 & 2 & 3,621 & 34 \\
\hline Selección raza local $\mathrm{N}^{\circ}$ & 28 & 4 & 1 & 3,619 & 35 \\
\hline Selección raza local $\mathrm{N}^{\circ}$ & 7 & 5 & 1 & 3,618 & 36 \\
\hline Selección raza local $\mathrm{N}^{\circ}$ & 91 & 3 & 2 & 3,618 & 37 \\
\hline Selección raza local $\mathrm{N}^{\circ}$ & 91 & 3 & 4 & 3,582 & 38 \\
\hline Selección raza local $\mathrm{N}^{\circ}$ & 74 & 5 & 1 & 3,459 & 39 \\
\hline Selección raza local $N^{\circ}$ & 20 & 1 & 3 & 3,406 & 40 \\
\hline Madre australiana en $\mathrm{HSC} \mathrm{N}^{\circ}$ & $\frac{2}{2}$ & 1 & 4 & 3,379 & 41 \\
\hline Madre australiana en $\mathrm{HSC} \mathrm{N}^{\circ}$ & 15 & 5 & 4 & 3,379 & 42 \\
\hline Selección raza local $N^{\circ}$ & 7 & 3 & 1 & 3,356 & 43 \\
\hline Selección raza local $\mathrm{N}^{\circ}$ & 91 & 4 & 1 & 3,345 & 44 \\
\hline Selección raza local $\mathrm{N}^{\circ}$ & 36 & 1 & 3 & 3,340 & 45 \\
\hline Selección raza local $N^{\circ}$ & 33 & 3 & 3 & 3,228 & 46 \\
\hline Madre australiana en $\mathrm{HSC} \mathrm{N}^{\circ}$ & 2 & 1 & 1 & 3,228 & 47 \\
\hline Selección raza local $N^{\circ}$ & 104 & 1 & 4 & 3,193 & 48 \\
\hline Selección raza local $\mathrm{N}^{\circ}$ & 104 & 5 & 4 & 3,081 & 49 \\
\hline Selección raza local $\mathrm{N}^{\circ}$ & 66 & 1 & 3 & 3,035 & 50 \\
\hline Madre australiana en $\mathrm{HSC} \mathrm{N}^{\circ}$ & 2 & 6 & 3 & 3,031 & 51 \\
\hline Selección raza local $N^{\circ}$ & 73 & 2 & 1 & 2,996 & 52 \\
\hline Selección raza local $N^{\circ}$ & 106 & 4 & 1 & 2,993 & 53 \\
\hline Madre australiana en $\mathrm{HSC} \mathrm{N}^{\circ}$ & 15 & 5 & 1 & 2,981 & 54 \\
\hline Selección raza local $N^{\circ}$ & 57 & 1 & 1 & 2,972 & 55 \\
\hline Selección raza local $\mathrm{N}^{\circ}$ & 57 & 6 & 1 & 2,970 & 56 \\
\hline Selección raza local $\mathrm{N}^{\circ}$ & 106 & 2 & 3 & 2,947 & 57 \\
\hline Selección raza local $\mathrm{N}^{\circ}$ & 74 & 2 & 3 & 2,936 & 58 \\
\hline Selección raza local $N^{\circ}$ & 79 & 1 & 3 & 2,936 & 59 \\
\hline Selección raza local $\mathrm{N}^{\circ}$ & 27 & 1 & 4 & 2,935 & 60 \\
\hline
\end{tabular}


APÉNDICE 2

RANKING FAMILIAR ENSAYO LA POSADA

\begin{tabular}{|c|c|c|c|}
\hline Madre & & Índice & Ranking \\
\hline Selección raza local $N^{\circ}$ & 57 & 2,67180217 & 1 \\
\hline Selección raza local $N^{\circ}$ & 104 & 2,52014762 & 2 \\
\hline Selección raza local Nº & 91 & 1,76639682 & 3 \\
\hline Selección raza local $N^{\circ}$ & 79 & 1,65484552 & 4 \\
\hline Selección raza local Nº & 106 & 1,42681611 & 5 \\
\hline Madre australiana en $\mathrm{HSC} \mathrm{N}^{\circ}$ & 6 & 1,4072 & 6 \\
\hline Selección raza local Nº & 73 & 0,98934691 & 7 \\
\hline Selección raza local $N^{\circ}$ & 28 & 0,97498714 & 8 \\
\hline Madre australiana en $\mathrm{HSC} \mathrm{N}^{\circ}$ & 33 & 0,96845824 & 9 \\
\hline Selección raza local $N^{\circ}$ & 42 & 0,96616132 & 10 \\
\hline Selección raza local Nº & 52 & 0,96334056 & 11 \\
\hline Selección raza local $N^{\circ}$ & 74 & 0,9587 & 12 \\
\hline Madre australiana en $\mathrm{HSC} \mathrm{N}^{\circ}$ & 2 & 0,84792555 & 13 \\
\hline Selección raza local $N^{\circ}$ & 36 & 0,74515261 & 14 \\
\hline Selección raza local $N^{\circ}$ & 7 & 0,74284205 & 15 \\
\hline Selección raza local $N^{\circ}$ & 27 & 0,68649739 & 16 \\
\hline Selección raza local $\mathrm{N}^{\circ}$ & 16 & 0,57372084 & 17 \\
\hline Selección raza local $N^{\circ}$ & 66 & 0,56876781 & 18 \\
\hline Madre australiana en $\mathrm{HSC} \mathrm{N}^{\circ}$ & 15 & 0,3723813 & 19 \\
\hline Selección raza local Nº & 22 & 0,35844634 & 20 \\
\hline Selección raza local $N^{\circ}$ & 25 & 0,30873333 & 21 \\
\hline Selección raza local $N^{\circ}$ & 14 & 0,21776542 & 22 \\
\hline Selección raza local $N^{\circ}$ & 12 & 0,21278558 & 23 \\
\hline Selección raza local $N^{\circ}$ & 20 & 0,15666133 & 24 \\
\hline Selección raza local $N^{\circ}$ & 41 & $-0,03982068$ & 25 \\
\hline Selección raza local Nº & 92 & $-0,1439769$ & 26 \\
\hline Madre australiana en $\mathrm{HSC} \mathrm{N}^{\circ}$ & 1 & $-0,22082875$ & 27 \\
\hline Selección raza local $N^{\circ}$ & 54 & $-0,32854167$ & 28 \\
\hline Selección raza local $N^{\circ}$ & 86 & $-0,33806455$ & 29 \\
\hline Selección raza local $N^{\circ}$ & 3 & $-0,35915706$ & 30 \\
\hline Madre australiana en $\mathrm{HSC} \mathrm{N}^{\circ}$ & 13 & $-0,3594$ & 31 \\
\hline Madre australiana en $\mathrm{HSC} \mathrm{N}^{\circ}$ & 16 & $-0,360625$ & 32 \\
\hline Selección raza local $\mathrm{N}^{\circ}$ & 39 & $-0,46962227$ & 33 \\
\hline Selección raza local $N^{\circ}$ & 30 & $-0,48094623$ & 34 \\
\hline Madre australiana en $\mathrm{HSC} \mathrm{N}^{\circ}$ & 7 & $-0,58022009$ & 35 \\
\hline Madre australiana en $\mathrm{HSC} \mathrm{N}^{\circ}$ & 11 & $-0,678755$ & 36 \\
\hline Selección raza local $\mathrm{N}^{\circ}$ & 40 & $-0,69787455$ & 37 \\
\hline Madre australiana en $\mathrm{HSC} \mathrm{N}^{\circ}$ & 25 & $-0,79013496$ & 38 \\
\hline Selección raza local $N^{\circ}$ & 107 & $-0,98591$ & 39 \\
\hline Selección raza local Nº & 105 & $-1,22748273$ & 40 \\
\hline Selección raza local Nº & 81 & $-1,265737$ & 41 \\
\hline Madre australiana en $\mathrm{HSC} \mathrm{N}^{\circ}$ & 26 & $-1,43655048$ & 42 \\
\hline Selección raza local $N^{\circ}$ & 96 & $-1,882925$ & 43 \\
\hline E. cladocalyx (control) & & $-2,80899524$ & 44 \\
\hline Selección raza local $\mathrm{N}^{\circ}$ & 69 & $-3,05135909$ & 45 \\
\hline E. camaldulensis (control) & & $-5,05761905$ & 46 \\
\hline
\end{tabular}


APÉNDICE $N^{\circ} 3$

RANKING DE ÁRBOLES INDIVIDUALES DEL ENSAYO SAN VICENTE (Primer $5 \%$ del ranking)

\begin{tabular}{|c|c|c|c|c|c|}
\hline Madre & & Bloque & Árbol & Índice & Ranking \\
\hline Madre australiana en $\mathrm{HSC} \mathrm{N}^{\circ}$ & 115 & 2 & 3 & 5,5262057 & 1 \\
\hline Selección raza local Nº & 241 & 2 & 4 & 5,2254703 & 2 \\
\hline Selección raza local $\mathrm{N}^{\circ}$ & 266 & 1 & 4 & 4,994102 & 3 \\
\hline Selección raza local $\mathrm{N}^{\circ}$ & 291 & 1 & 1 & 4,6599186 & 4 \\
\hline Selección raza local Nº & 273 & 2 & 4 & 4,4802804 & 5 \\
\hline Madre australiana en $\mathrm{HSC} \mathrm{N}^{\circ}$ & 17 & 2 & 2 & 4,4187061 & 6 \\
\hline Selección raza local N & 27 & 2 & 1 & 4,343686 & 7 \\
\hline Selección raza local Nº & 273 & 6 & 2 & 4,2839837 & 8 \\
\hline Selección raza local $\mathrm{N}^{\circ}$ & 212 & 2 & 3 & 4,1811061 & 9 \\
\hline Selección raza local $\mathrm{N}^{\circ}$ & 294 & 4 & 4 & 4,1448467 & 10 \\
\hline Madre australiana en $\mathrm{HSC} \mathrm{N}^{\circ}$ & 125 & 2 & 4 & 4,087858 & 11 \\
\hline Madre australiana en $\mathrm{HSC} \mathrm{N}^{\circ}$ & 17 & 4 & 1 & 4,0411099 & 12 \\
\hline Madre australiana en $\mathrm{HSC} \mathrm{N}^{\circ}$ & 16 & 2 & 1 & 3,8973849 & 13 \\
\hline Selección raza local Nº & 2106 & 1 & 3 & 3,8590675 & 14 \\
\hline Selección raza local $\mathrm{N}^{\circ}$ & 294 & 5 & 2 & 3,8135566 & 15 \\
\hline Selección raza local No & 227 & 3 & 4 & 3,7985884 & 16 \\
\hline Madre australiana en $\mathrm{HSC} \mathrm{N}^{\circ}$ & 115 & 3 & 4 & 3,7740577 & 17 \\
\hline Madre australiana en $\mathrm{HSC} \mathrm{N}^{\circ}$ & 115 & 2 & 1 & 3,7443568 & 18 \\
\hline Madre australiana en $\mathrm{HSC} \mathrm{N}^{\circ}$ & 12 & 5 & 3 & 3,7346548 & 19 \\
\hline Selección raza local Nº & 279 & 5 & 4 & 3,6344548 & 20 \\
\hline Selección raza local № & 2106 & 3 & 3 & 3,5805051 & 21 \\
\hline Selección raza local № & 2104 & 2 & 3 & 3,5642643 & 22 \\
\hline Selección raza local $\mathrm{N}^{\circ}$ & 291 & 3 & 1 & 3,5577056 & 23 \\
\hline Selección raza local № & 212 & 2 & 4 & 3,5524695 & 24 \\
\hline Selección raza local № & 294 & 5 & 4 & 3,5419592 & 25 \\
\hline Madre australiana en $\mathrm{HSC} \mathrm{N}^{\circ}$ & 17 & 2 & 1 & 3,541004 & 26 \\
\hline Selección raza local No ${ }^{\circ}$ & 291 & 5 & 4 & 3,4223789 & 27 \\
\hline Selección raza local $\mathrm{N}^{\circ}$ & 291 & 5 & 3 & 3,3476499 & 28 \\
\hline Selección raza local $\mathrm{N}^{\circ}$ & 291 & 5 & 1 & 3,3347144 & 29 \\
\hline Selección raza local $\mathrm{N}^{\circ}$ & 2104 & 2 & 1 & 3,328712 & 30 \\
\hline Selección raza local Nº & 2104 & 1 & 2 & 3,2819972 & 31 \\
\hline Madre australiana en $\mathrm{HSC} \mathrm{N}^{\circ}$ & 133 & 4 & 2 & 3,2647931 & 32 \\
\hline Madre australiana en $\mathrm{HSC} \mathrm{N}^{\circ}$ & 115 & 5 & 2 & 3,2594481 & 33 \\
\hline Selección raza local N ${ }^{\circ}$ & 273 & 4 & 3 & 3,2156739 & 34 \\
\hline Madre australiana en $\mathrm{HSC} \mathrm{N}^{\circ}$ & 17 & 4 & 4 & 3,186644 & 35 \\
\hline Madre australiana en $\mathrm{HSC} \mathrm{N}^{\circ}$ & 17 & 5 & 4 & 3,1697136 & 36 \\
\hline Madre australiana en $\mathrm{HSC} \mathrm{N}^{\circ}$ & 133 & 3 & 2 & 3,1650193 & 37 \\
\hline Selección raza local № & 279 & 2 & 2 & 3,1458441 & 38 \\
\hline Selección raza local № & 294 & 3 & 3 & 3,130752 & 39 \\
\hline Selección raza local № & 216 & 3 & 4 & 3,1002577 & 40 \\
\hline Selección raza local N ${ }^{\circ}$ & 291 & 1 & 2 & 3,0709863 & 41 \\
\hline Madre australiana en $\mathrm{HSC} \mathrm{N}^{\circ}$ & 11 & 4 & 2 & 3,0439974 & 42 \\
\hline Selección raza local Nº & 254 & 5 & 1 & 3,0331768 & 43 \\
\hline Selección raza local $\mathrm{N}^{\circ}$ & 216 & 3 & 3 & 3,0325341 & 44 \\
\hline Selección raza local Nº & 294 & 3 & 2 & 3,0316354 & 45 \\
\hline Madre australiana en $\mathrm{HSC} \mathrm{N}^{\circ}$ & 16 & 3 & 1 & 3,0311356 & 46 \\
\hline Selección raza local N ${ }^{\circ}$ & 252 & 3 & 3 & 3,0063064 & 47 \\
\hline Selección raza local $N^{\circ}$ & 273 & 6 & 1 & 2,9915313 & 48 \\
\hline Madre australiana en $\mathrm{HSC} \mathrm{N}^{\circ}$ & 115 & 3 & 2 & 2,9486315 & 49 \\
\hline Selección raza local $\mathrm{N}^{\circ}$ & 267 & 3 & 2 & 2,9390835 & 50 \\
\hline Madre australiana en $\mathrm{HSC} \mathrm{N}^{\circ}$ & 125 & 3 & 3 & 2,9332856 & 51 \\
\hline Madre australiana en $\mathrm{HSC} \mathrm{N}^{\circ}$ & 12 & 2 & 2 & 2,9201885 & 52 \\
\hline Madre australiana en $\mathrm{HSC} \mathrm{N}^{\circ}$ & 17 & 4 & 2 & 2,9145517 & 53 \\
\hline Selección raza local № & 266 & 6 & 3 & 2,8448331 & 54 \\
\hline Madre australiana en $\mathrm{HSC} \mathrm{N}^{\circ}$ & 116 & 3 & 3 & 2,8400611 & 55 \\
\hline Madre australiana en $\mathrm{HSC} \mathrm{N}^{\circ}$ & 214 & 3 & 4 & 2,8279951 & 56 \\
\hline Selección raza local № & 214 & 3 & 2 & 2,8246998 & 57 \\
\hline Selección raza local № & 288 & 5 & 4 & 2,8155485 & 58 \\
\hline Madre australiana en $\mathrm{HSC} \mathrm{N}^{\circ}$ & 111 & 3 & 2 & 2,8108821 & 59 \\
\hline Madre australiana en $\mathrm{HSC} \mathrm{N}^{\circ}$ & 115 & 5 & 3 & 2,7889172 & 60 \\
\hline
\end{tabular}


APÉNDICE 4

RANKING FAMILIAR ENSAYO SAN VICENTE

\begin{tabular}{|c|c|c|}
\hline Madre & Índice & Ranking \\
\hline Madre australiana en HSC 7 & 2,25565915 & 1 \\
\hline Selección raza local 73 & 2,20547693 & 2 \\
\hline Selección raza local 91 & 1,7056267 & 3 \\
\hline Madre australiana en HSC 2 & 1,54351931 & 4 \\
\hline Madre australiana en HSC 15 & 1,46626584 & 5 \\
\hline Madre australiana en HSC 25 & 1,41617116 & 6 \\
\hline Selección raza local 94 & 1,3202014 & 7 \\
\hline Selección raza local 106 & 1,29572023 & 8 \\
\hline Selección raza local 104 & 1,12211162 & 9 \\
\hline Selección raza local 27 & 1,03146224 & 10 \\
\hline Madre australiana en HSC 33 & 1,00562934 & 11 \\
\hline Madre australiana en HSC 26 & 0,82656977 & 12 \\
\hline Selección raza local 57 & 0,77506622 & 13 \\
\hline Selección raza local 79 & 0,62293193 & 14 \\
\hline Selección raza local 56 & 0,60618136 & 15 \\
\hline Selección raza local 61 & 0,559407 & 16 \\
\hline Selección raza local 101 & 0,54913276 & 17 \\
\hline Selección raza local 41 & 0,54256724 & 18 \\
\hline Madre australiana en HSC 6 & 0,48619052 & 19 \\
\hline Selección raza local 36 & 0,47833677 & 20 \\
\hline Selección raza local 74 & 0,46221787 & 21 \\
\hline Selección raza local 67 & 0,41436574 & 22 \\
\hline Madre australiana en HSC 16 & 0,36010013 & 23 \\
\hline Madre australiana en HSC 5 & 0,33917801 & 24 \\
\hline Selección raza local 14 & 0,32443498 & 25 \\
\hline Selección raza local 12 & 0,31361742 & 26 \\
\hline Selección raza local 7 & 0,27319644 & 27 \\
\hline Selección raza local 96 & 0,26276717 & 28 \\
\hline Selección raza local 66 & 0,25413019 & 29 \\
\hline Selección raza local 34 & 0,23529513 & 30 \\
\hline Selección raza local 5 & 0,12056915 & 31 \\
\hline Selección raza local 30 & 0,094049 & 32 \\
\hline Selección raza local 16 & 0,09202543 & 33 \\
\hline Selección raza local 88 & 0,03523804 & 34 \\
\hline Selección raza local 52 & $-0,0420195$ & 35 \\
\hline Madre australiana en HSC 1 & $-0,0464149$ & 36 \\
\hline Selección raza local 15 & $-0,21443658$ & 37 \\
\hline Selección raza local 42 & $-0,29089678$ & 38 \\
\hline Selección raza local 20 & $-0,2950097$ & 39 \\
\hline Selección raza local 81 & $-0,30946729$ & 40 \\
\hline Selección raza local 107 & $-0,41292309$ & 41 \\
\hline Selección raza local 39 & $-0,45366593$ & 42 \\
\hline Selección raza local 28 & $-0,45536621$ & 43 \\
\hline Selección raza local 92 & $-0,47506363$ & 44 \\
\hline Selección raza local 3 & $-0,49361041$ & 45 \\
\hline Selección raza local 29 & $-0,51230091$ & 46 \\
\hline Madre australiana en HSC 11 & $-0,53235132$ & 47 \\
\hline Selección raza local 86 & $-0,57353166$ & 48 \\
\hline Selección raza local 23 & $-0,57906364$ & 49 \\
\hline Selección raza local 22 & $-0,6410808$ & 50 \\
\hline Selección raza local 54 & $-0,72671268$ & 51 \\
\hline Selección raza local 71 & $-0,76747157$ & 52 \\
\hline Selección raza local 89 & $-0,92353819$ & 53 \\
\hline Selección raza local 40 & $-1,02160338$ & 54 \\
\hline Selección raza local 69 & $-1,12285684$ & 55 \\
\hline Selección raza local 100 & $-1,17628431$ & 56 \\
\hline Madre australiana en HSC 13 & $-1,21797325$ & 57 \\
\hline Selección raza local 105 & $-1,26816508$ & 58 \\
\hline Selección raza local 24 & $-1,34705699$ & 59 \\
\hline Selección raza local 37 & $-1,47643352$ & 60 \\
\hline E. cladocalyx (control) & $-2,53366003$ & 61 \\
\hline E. camaldulensis (control) & $-2,59086234$ & 62 \\
\hline Selección raza local 26 & $-2,85304116$ & 63 \\
\hline
\end{tabular}




\section{APÉNDICE N ${ }^{\circ} 5$ \\ RANKING DE ÁRBOLES INDIVIDUALES DEL ENSAYO EL MIRADOR (Primer 5\% del ranking)}

\begin{tabular}{|c|c|c|c|c|c|c|c|}
\hline Madre & & Padre & & Bloque & Árbol & Índice & Ranking \\
\hline Madre australiana en $\mathrm{HSC} \mathrm{N}^{\circ}$ & 40 & Selección raza local $N^{\circ}$ & 72 & 4 & 1 & 1,8215509 & 1 \\
\hline Madre australiana en $\mathrm{HSC} \mathrm{N}^{\circ}$ & 40 & Selección raza local № & 72 & 2 & 4 & 1,7878094 & 2 \\
\hline Madre australiana en $\mathrm{HSC} \mathrm{N}^{\circ}$ & 40 & Selección raza local № & 72 & 4 & 2 & 1,7415281 & 3 \\
\hline Madre australiana en $\mathrm{HSC} \mathrm{N}^{\circ}$ & 40 & Selección raza local № & 72 & 3 & 1 & 1,5995291 & 4 \\
\hline Madre australiana en $\mathrm{HSC} \mathrm{N}^{\circ}$ & 40 & Selección raza local № & 72 & 4 & 3 & 1,4938209 & 5 \\
\hline Madre australiana en $\mathrm{HSC} \mathrm{N}^{\circ}$ & 40 & Selección raza local № & 72 & 3 & 4 & 1,4197365 & 6 \\
\hline Madre australiana en $\mathrm{HSC} \mathrm{N}^{\circ}$ & 5 & Selección raza local № & 70 & 2 & 1 & 1,3772993 & 7 \\
\hline Madre australiana en $\mathrm{HSC} \mathrm{N}^{\circ}$ & 15 & Selección raza local $N^{\circ}$ & 70 & 2 & 3 & 1,3674557 & 8 \\
\hline Madre australiana en $\mathrm{HSC} \mathrm{N}^{\circ}$ & 5 & Selección raza local № & 72 & 2 & 1 & 1,3513942 & 9 \\
\hline Madre australiana en $\mathrm{HSC} \mathrm{N}^{\circ}$ & 5 & Polinización abierta & & 4 & 2 & 1,3333151 & 10 \\
\hline Madre australiana en $\mathrm{HSC} \mathrm{N}^{\circ}$ & 15 & Polinización abierta & & 2 & 1 & 1,3176111 & 11 \\
\hline Madre australiana en $\mathrm{HSC} \mathrm{N}^{\circ}$ & 7 & Polinización abierta & & 6 & 3 & 1,2971283 & 12 \\
\hline Madre australiana en $\mathrm{HSC} \mathrm{N}^{\circ}$ & 15 & Selección raza local № & 70 & 1 & 4 & 1,2888057 & 13 \\
\hline Madre australiana en $\mathrm{HSC} \mathrm{N}^{\circ}$ & 15 & Selección raza local № & 70 & 1 & 4 & 1,2786163 & 14 \\
\hline Madre australiana en $\mathrm{HSC} \mathrm{N}^{\circ}$ & 5 & Selección raza local № & 70 & 1 & 1 & 1,2403794 & 15 \\
\hline Madre australiana en $\mathrm{HSC} \mathrm{N}^{\circ}$ & 40 & Selección raza local № & 72 & 3 & 2 & 1,2365522 & 16 \\
\hline Madre australiana en $\mathrm{HSC} \mathrm{N}^{\circ}$ & 5 & Selección raza local № & 70 & 1 & 2 & 1,2356149 & 17 \\
\hline Madre australiana en $\mathrm{HSC} \mathrm{N}^{\circ}$ & 40 & Selección raza local № & 72 & 4 & 4 & 1,2255789 & 18 \\
\hline Madre australiana en $\mathrm{HSC} \mathrm{N}^{\circ}$ & 5 & Selección raza local № & 72 & 2 & 3 & 1,2157249 & 19 \\
\hline Madre australiana en $\mathrm{HSC} \mathrm{N}^{\circ}$ & 5 & Selección raza local № & 72 & 3 & 2 & 1,1949197 & 20 \\
\hline Madre australiana en $\mathrm{HSC} \mathrm{N}^{\circ}$ & 40 & Polinización abierta & & 4 & 1 & 1,1767015 & 21 \\
\hline Madre australiana en $\mathrm{HSC} \mathrm{N}^{\circ}$ & 15 & Selección raza local № & 70 & 3 & 3 & 1,128114 & 22 \\
\hline Madre australiana en $\mathrm{HSC} \mathrm{N}^{\circ}$ & 5 & Selección raza local № & 72 & 3 & 4 & 1,127321 & 23 \\
\hline Madre australiana en $\mathrm{HSC} \mathrm{N}^{\circ}$ & 15 & Selección raza local № & 70 & 1 & 2 & 1,111656 & 24 \\
\hline Madre australiana en $\mathrm{HSC} \mathrm{N}^{\circ}$ & 5 & Selección raza local № & 70 & 3 & 4 & 1,0863307 & 25 \\
\hline Madre australiana en $\mathrm{HSC} \mathrm{N}^{\circ}$ & 32 & Selección raza local № & 22 & 3 & 2 & 1,0673663 & 26 \\
\hline Madre australiana en $\mathrm{HSC} \mathrm{N}^{\circ}$ & 32 & Mezcla polen raza local & & 4 & 4 & 1,0496005 & 27 \\
\hline Madre australiana en $\mathrm{HSC} \mathrm{N}^{\circ}$ & 40 & Polinización abierta & & 3 & 1 & 1,0262551 & 28 \\
\hline Madre australiana en $\mathrm{HSC} \mathrm{N}^{\circ}$ & 15 & Polinización abierta & & 6 & 4 & 0,9930375 & 29 \\
\hline Madre australiana en $\mathrm{HSC} \mathrm{N}^{\circ}$ & 15 & Selección raza local № & 70 & 3 & 3 & 0,9862554 & 30 \\
\hline Madre australiana en $\mathrm{HSC} \mathrm{N}^{\circ}$ & 15 & Selección raza local № & 70 & 6 & 1 & 0,9732307 & 31 \\
\hline Madre australiana en $\mathrm{HSC} \mathrm{N}^{\circ}$ & 5 & Selección raza local № & 83 & 6 & 2 & 0,958893 & 32 \\
\hline Madre australiana en $\mathrm{HSC} \mathrm{N}^{\circ}$ & 5 & Selección raza local № & 22 & 2 & 3 & 0,9510969 & 33 \\
\hline Madre australiana en $\mathrm{HSC} \mathrm{N}^{\circ}$ & 40 & Selección raza local № & 72 & 3 & 3 & 0,9488349 & 34 \\
\hline Madre australiana en $\mathrm{HSC} \mathrm{N}^{\circ}$ & 5 & Selección raza local № & 70 & 2 & 4 & 0,9465664 & 35 \\
\hline Madre australiana en $\mathrm{HSC} \mathrm{N}^{\circ}$ & 5 & Selección raza local № & 22 & 2 & 4 & 0,943436 & 36 \\
\hline Madre australiana en $\mathrm{HSC} \mathrm{N}^{\circ}$ & 33 & Polinización abierta & & 6 & 3 & 0,93613 & 37 \\
\hline Madre australiana en $\mathrm{HSC} \mathrm{N}^{\circ}$ & 32 & Selección raza local № & 22 & 6 & 2 & 0,9264905 & 38 \\
\hline Madre australiana en $\mathrm{HSC} \mathrm{N}^{\circ}$ & 15 & Selección raza local $\mathrm{N}^{\circ}$ & 70 & 2 & 2 & 0,923533 & 39 \\
\hline Madre australiana en $\mathrm{HSC} \mathrm{N}^{\circ}$ & 5 & Selección raza local № & 70 & 3 & 1 & 0,9214491 & 40 \\
\hline Madre australiana en $\mathrm{HSC} \mathrm{N}^{\circ}$ & 5 & Polinización abierta & & 4 & 3 & 0,9212606 & 41 \\
\hline Madre australiana en $\mathrm{HSC} \mathrm{N}^{\circ}$ & 40 & Polinización abierta & & 2 & 3 & 0,9208979 & 42 \\
\hline Madre australiana en $\mathrm{HSC} \mathrm{N}^{\circ}$ & 40 & Selección raza local № & 72 & 2 & 1 & 0,9138571 & 43 \\
\hline Madre australiana en $\mathrm{HSC} \mathrm{N}^{\circ}$ & 5 & Selección raza local № & 72 & 2 & 2 & 0,913705 & 44 \\
\hline Madre australiana en $\mathrm{HSC} \mathrm{N}^{\circ}$ & 15 & Selección raza local № & 70 & 3 & 4 & 0,9129172 & 45 \\
\hline
\end{tabular}




\section{APÉNDICE 6 \\ RANKING FAMILIAR POR CRUZA ENSAYO EL MIRADOR (Aptitud combinatoria específica de las cruzas)}

\begin{tabular}{|c|c|c|c|c|c|c|}
\hline Cruza & Madre & & Padre & & Índice & Ranking \\
\hline 228 & Madre australiana en $\mathrm{HSC} \mathrm{N}^{\circ}$ & 40 & Selección raza local № & 72 & 1,26750978 & 1 \\
\hline 23 & Madre australiana en $\mathrm{HSC} \mathrm{N}^{\circ}$ & 5 & Selección raza local $\mathrm{N}^{\circ}$ & 72 & 1,09328489 & 2 \\
\hline 22 & Madre australiana en $\mathrm{HSC} \mathrm{N}^{\circ}$ & 5 & Selección raza local $\mathrm{N}^{\circ}$ & 70 & 0,67284127 & 3 \\
\hline 211 & Madre australiana en $\mathrm{HSC} \mathrm{N}^{\circ}$ & 15 & Selección raza local $N^{\circ}$ & 70 & 0,65257272 & 4 \\
\hline 140 & Madre australiana en $\mathrm{HSC} \mathrm{N}^{\circ}$ & 40 & Polinización abierta & & 0,59619022 & 5 \\
\hline 21 & Madre australiana en $\mathrm{HSC} \mathrm{N}^{\circ}$ & 5 & Selección raza local $N^{\circ}$ & 22 & 0,53436903 & 6 \\
\hline 15 & Madre australiana en $\mathrm{HSC} \mathrm{N}^{\circ}$ & 5 & Polinización abierta & & 0,49727608 & 7 \\
\hline 220 & Madre australiana en $\mathrm{HSC} \mathrm{N}^{\circ}$ & 32 & Selección raza local $N^{\circ}$ & 22 & 0,4783704 & 8 \\
\hline 221 & Madre australiana en $\mathrm{HSC} \mathrm{N}^{\circ}$ & 32 & Selección raza local $N^{\circ}$ & 70 & 0,45495939 & 9 \\
\hline 226 & Madre australiana en $\mathrm{HSC} \mathrm{N}^{\circ}$ & 32 & Mezcla polen raza local & & 0,35488413 & 10 \\
\hline 29 & Madre australiana en $\mathrm{HSC} \mathrm{N}^{\circ}$ & 7 & Selección raza local $N^{\circ}$ & 70 & 0,33170001 & 11 \\
\hline 229 & Madre australiana en $\mathrm{HSC} \mathrm{N}^{\circ}$ & 40 & Selección raza local $N^{\circ}$ & 83 & 0,28828697 & 12 \\
\hline 666 & \multicolumn{4}{|c|}{ E. globulus (control) } & 0,28706452 & 13 \\
\hline 115 & Madre australiana en $\mathrm{HSC} \mathrm{N}^{\circ}$ & 15 & Polinización abierta & & 0,27002784 & 14 \\
\hline 25 & Madre australiana en $\mathrm{HSC} \mathrm{N}^{\circ}$ & 5 & Selección raza local $N^{\circ}$ & 83 & 0,26955108 & 15 \\
\hline 222 & Madre australiana en $\mathrm{HSC} \mathrm{N}^{\circ}$ & 32 & Selección raza local N & 80 & 0,23165309 & 16 \\
\hline 223 & Madre australiana en $\mathrm{HSC} \mathrm{N}^{\circ}$ & 32 & Selección raza local $N^{\circ}$ & 83 & 0,21151557 & 17 \\
\hline 24 & Madre australiana en $\mathrm{HSC} \mathrm{N}^{\circ}$ & 5 & Selección raza local $N^{\circ}$ & 80 & 0,1957799 & 18 \\
\hline 27 & Madre australiana en $\mathrm{HSC} \mathrm{N}^{\circ}$ & 5 & Selección raza local $N^{\circ}$ & 89 & 0,17995727 & 19 \\
\hline 225 & Madre australiana en $\mathrm{HSC}^{\circ}$ & 32 & Selección raza local $N^{\circ}$ & 89 & 0,15535348 & 20 \\
\hline 215 & Madre australiana en $\mathrm{HSC} \mathrm{N}^{\circ}$ & 15 & Mezcla polen raza local & & 0,15510674 & 21 \\
\hline 26 & Madre australiana en $\mathrm{HSC} \mathrm{N}^{\circ}$ & 5 & Selección raza local $N^{\circ}$ & 86 & 0,12128858 & 22 \\
\hline 216 & Madre australiana en $\mathrm{HSC} \mathrm{N}^{\circ}$ & 26 & Selección raza local $N^{\circ}$ & 70 & 0,02957803 & 23 \\
\hline 17 & Madre australiana en $\mathrm{HSC} \mathrm{N}^{\circ}$ & 7 & Polinización abierta & & $-0,00321343$ & 24 \\
\hline 210 & Madre australiana en $\mathrm{HSC} \mathrm{N}^{\circ}$ & 7 & Selección raza local $N^{\circ}$ & 80 & $-0,09543659$ & 25 \\
\hline 224 & Madre australiana en $\mathrm{HSC}^{\circ}$ & 32 & Selección raza local $N^{\circ}$ & 86 & $-0,11182355$ & 26 \\
\hline 219 & Madre australiana en $\mathrm{HSC} \mathrm{N}^{\circ}$ & 26 & Mezcla polen raza local & & $-0,12369818$ & 27 \\
\hline 126 & Madre australiana en $\mathrm{HSC} \mathrm{N}^{\circ}$ & 26 & Polinización abierta & & $-0,16932153$ & 28 \\
\hline 218 & Madre australiana en $\mathrm{HSC} \mathrm{N}^{\circ}$ & 26 & Selección raza local $N^{\circ}$ & 83 & $-0,21379001$ & 29 \\
\hline 133 & Madre australiana en $\mathrm{HSC} \mathrm{N}^{\circ}$ & 33 & Polinización abierta & & $-0,39014624$ & 30 \\
\hline 217 & Madre australiana en $\mathrm{HSC} \mathrm{N}^{\circ}$ & 26 & Selección raza local $N^{\circ}$ & 80 & $-0,40023289$ & 31 \\
\hline 444 & \multicolumn{4}{|c|}{ E. camaldulensis (control) } & $-1,47092108$ & 32 \\
\hline
\end{tabular}




\section{APÉNDICE 7}

RANKING FAMILIAR POR MADRE ENSAYO EL MIRADOR

(Aptitud combinatoria general madres)

\begin{tabular}{|l|c|c|}
\hline Madre & Índice & Ranking \\
\hline Madre australiana en HSC N ${ }^{\circ} 40$ & 0,71732899 & $\mathbf{1}$ \\
\hline Madre australiana en HSC N ${ }^{\circ} 15$ & 0,50558665 & $\mathbf{2}$ \\
\hline Madre australiana en HSC N 5 & 0,39484482 & $\mathbf{3}$ \\
\hline E. globulus (control) & 0,28706452 & $\mathbf{4}$ \\
\hline Madre australiana en HSC N ${ }^{\circ} 32$ & 0,25437718 & $\mathbf{5}$ \\
\hline Madre australiana en HSC N 7 & 0,07768333 & $\mathbf{6}$ \\
\hline Madre australiana en HSC N 26 & $-0,17549291$ & $\mathbf{7}$ \\
\hline Madre australiana en HSC N 33 & $-0,39014624$ & $\mathbf{8}$ \\
\hline E.camaldulensis (control) & $-1,47092108$ & $\mathbf{9}$ \\
\hline
\end{tabular}

\section{APÉNDICE 8}

RANKING FAMILIAR POR PADRE ENSAYO EL MIRADOR

(Aptitud combinatoria general padres)

\begin{tabular}{|c|c|c|}
\hline Padre & Índice & Ranking \\
\hline Selección raza local N 72 & 1,22395356 & 1 \\
\hline Selección raza local $N^{\circ} 22$ & 0,50636971 & 2 \\
\hline Selección raza local $N^{\circ} 70$ & 0,48641775 & 3 \\
\hline Selección raza local № 89 & 0,16791712 & 4 \\
\hline Selección raza local № 83 & 0,16502294 & 5 \\
\hline Mezcla polen raza local & 0,13550761 & 6 \\
\hline Selección raza local N 80 & 0,0184712 & 7 \\
\hline Selección raza local № 86 & 0,00473251 & 8 \\
\hline Polinizac abierta HSC & $-0,07699816$ & 9 \\
\hline
\end{tabular}


